\title{
Philosophiques
}

\section{Réduction fonctionnelle et réduction logique}

\section{Max Kistler}

Volume 27, numéro 1, printemps 2000

Le matérialisme contemporain

URI : https://id.erudit.org/iderudit/004938ar

DOI : https://doi.org/10.7202/004938ar

Aller au sommaire du numéro

\section{Éditeur(s)}

Société de philosophie du Québec

ISSN

0316-2923 (imprimé)

1492-1391 (numérique)

Découvrir la revue

Citer cet article

Kistler, M. (2000). Réduction fonctionnelle et réduction logique. Philosophiques, 27(1), 27-38. https://doi.org/10.7202/004938ar

\section{Résumé de l'article}

Kim attribue aux émergentistes un modèle de « réduction logique » dans lequel la prédiction ou l'explication d'une occurrence de la propriété réduite ne requiert, outre des informations sur le niveau réducteur, que des principes logiques et mathématiques. Sur la base de cette interprétation, je conteste deux thèses de Kim. La première concerne la légitimité du modèle émergentiste de réduction. J'essaie de montrer, à l'aide de l'exemple de l'addition des masses, que l'adoption de la réduction logique rendrait irréductibles certaines propriétés qui sont clairement réductibles. La deuxième est la thèse selon laquelle la réduction fonctionnelle correspond aux exigences émergentistes sur la réduction. Telle que Kim la caractérise, la réduction fonctionnelle comporte, outre une définition fonctionnelle de la propriété à réduire, l'indication des propriétés réalisatrices. Or cette information, qui correspond à la découverte d'une loi de correspondance (locale), est empirique et non seulement logique. 


\title{
Réduction fonctionnelle et réduction logique
}

\author{
MAX KISTLER \\ kistler@ext.jussieu.fr \\ Département de Philosophie \\ Université Paris X - Nanterre
}

\begin{abstract}
RÉSUMÉ. - Kim attribue aux émergentistes un modèle de « réduction logique » dans lequel la prédiction ou l'explication d'une occurrence de la propriété réduite ne requiert, outre des informations sur le niveau réducteur, que des principes logiques et mathématiques. Sur la base de cette interprétation, je conteste deux thèses de Kim. La première concerne la légitimité du modèle émergentiste de réduction. J'essaie de montrer, à l'aide de l'exemple de l'addition des masses, que l'adoption de la réduction logique rendrait irréductibles certaines propriétés qui sont clairement réductibles. La deuxième est la thèse selon laquelle la réduction fonctionnelle correspond aux exigences émergentistes sur la réduction. Telle que Kim la caractérise, la réduction fonctionnelle comporte, outre une définition fonctionnelle de la propriété à réduire, l'indication des propriétés réalisatrices. Or cette information, qui correspond à la découverte d'une loi de correspondance (locale), est empirique et non seulement logique.
\end{abstract}

\begin{abstract}
I read Kim as attributing to the emergentists a model of "logical reduction" in which the prediction or explanation of an occurrence of the reduced property requires, beyond information on the reducing level, only logi$\mathrm{cal}$ and mathematical principles. On the basis of this interpretation, I argue against two theses of Kim's. The first concerns the legitimacy of the emergentist model of reduction. I try to show by the example of the addition of masses that according to logical reduction certain properties which are clearly reducible come out as irreducible. The second is the thesis that functional reduction as construed by Kim satisfies the emergentist constraints on reduction. Functional reduction implies, over and above a functional definition of the reduced property, the indication of its realizers. But this information corresponding to the discovery of a (local) bridge law is empirical and not only logical.
\end{abstract}

D ans son texte récent « Émergence, modèles de réduction et le mental ${ }^{1}{ }$, Kim ne nous offre pas moins qu'une remise en cause radicale del'opinion reçue à la fois sur le modèle classique de la réduction proposé par $\mathrm{N}$ agel et sur les conséquences de la conception fonctionnaliste des états mentaux concernant leur capacité ou non à être réduits à des états cérébraux ${ }^{2}$. La conclusion du fameux argument dela réalisabilité multiple était qu'il est impossible de réduire les états mentaux à des états cérébraux. $D$ 'autre part, l'existence de cas de réduction réussie en l'absence de lois de correspondance (bridge laws) semblait montrer que le modèle nagelien était trop restrictif et qu'il fallait chercher des critères de réduction plus faibles que la découverte d'une loi de correspondance.

1. Voir l'article de Kim dans le présent numéro.

2. Pour le modèle classique de la réduction, voir $\mathrm{N}$ agel, 1961. 
Kim reconsidère ces arguments et parvient, dans les deux cas, à une conclusion inhabituelle. D'une part, il reconstruit un modèle de réduction qu'il attribue aux émergentistes du début du siècle et que j'appellerai le modèle de la « réduction logique ». Celui-ci impose des exigences plus fortes que celles du modèle nagelien, mais K im fait valoir que le modèle est plausible et correspond à des intuitions largement répandues, entre autres chez les scientifiques. Ce n'est pas que $\mathrm{N}$ agel soit trop exigeant, mais au contraire il ne le serait pas assez. D'autre part, contre la conviction répandue selon laquelle la conception fonctionnaliste des états mentaux entraîne leur irréductibilité, étant donné leur réalisabilité multiple, Kim élabore un concept de « réduction fonctionnelle » qui permet non seulement de montrer que le fonctionnal isme est compatible avec la réductibilité des états mentaux, mais qu'il s'agit d'une forme de réduction plus forte que celle du modèle nagelien : la réduction fonctionnelle serait une réduction logique au sens exigé par les émergentistes.

Il est toujours salutaire d'ébranler des vieilles certitudes. En ce sens, il faut saluer la démonstration de la modestie métaphysique de la réduction nagelienne qui la rend compatible avec des «théories dualistes classiques concernant la relation corps/esprit - comme par exemple la théorie spinoziste du double aspect, la doctrine de l'harmonie préétablie de Leibniz, le parallélisme ou le monisme neutre ${ }^{3} »$. II faut aussi reconnaître la plausibilité intuitive d'une doctrine de la réduction plus exigeante, comme celle de la réduction logique des émergentistes.

Dans ce qui suit, je conteste deux thèses cruciales avancées par Kim. La première concerne la légitimité du modèle émergentiste de réduction. J'essaie de montrer, en meservant del'exempledel'addition des masses, quel'adoption de la réduction logique rend émergentes, et donc irréductibles, certaines propriétés clairement réductibles. La deuxième est la thèse selon laquelle la réduction fonctionnelle satisferait aux exigences émergentistes sur la réduction.

\section{Le modèle émergentiste de la « réduction logique »}

Selon la reconstruction par Kim du concept de réduction employé par les émergentistes, une réduction nagelienne achevée ne constitue pas encore une réduction véritable, dans la mesure où ses prémisses ne font pas exclusivement référence aux propriétés et lois du niveau réducteur. La question-clé qui permet de distinguer les vraies réductions est la suivante : "L'occurrence d'une propriété de niveau supérieur peut-elle être prédite uniquement à partir d'informations concernant le niveau de base ${ }^{4}$ ? » Selon la réduction nagelienne, la réponse est non, car les lois de correspondance qui sont une composante essentielle de ses prémisses font référence à un lien entre propriétés

3. Voir Kim dans le présent numéro, p. 17

4. Ibid. 
réductrices et propriétés réduites. Dans la mesure où les lois de correspondance font référence aux propriétés réduites - de haut niveau - la prédiction que fournit une réduction nagelienne, en accord avec le modèle déductifnomologique (ou D-N), n'est pas basée sur la seule information concernant le niveau de base. K im montre que cette exigence est l'expression d'une intuition largement répandue sur ce qu'il faut demander à une véritable réduction. Entre autres, son inclusion dans une définition de la réduction permettrait de justifier l'intuition selon laquelle les dualismes spinoziste, leibnizien ou cartésien ne devraient pas compter parmi les théories réductionnistes. $O r$, comme ces conceptions impliquent l'existence de lois de correspondance, elles comptent comme réductionnistes selon le modèle nagelien.

$M$ ais une réduction sans lois de correspondance est-elle seulement envisageable? Si l'exigence émergentiste s'avérait trop forte pour être satisfaite, l'imposer serait un moyen de stipuler l'antiréductionnisme. M ais ce ne serait guère convaincant dans la mesure où cette conception déboucherait sur le résultat que toutes les propriétés macroscopiques sont irréductibles. $\mathrm{Or}$ une telle conclusion est inacceptable, étant donné qu'il existe au moins quelques cas non controversés de réduction réussie. L'intention des émergentistes eux-mêmes n'était pas de faire de toutes les propriétés macroscopiques des propriétés émergentes, mais au contraire d'opposer une classe particulière de propriétés émergentes à une autre classe, probablement plus vaste, de propriétés « résultantes ». Pour ces dernières, ils réclament la possibilitéque leur occurrence, contrairement à celle des propriétés émergentes, puisse être prédite sur la seule base d'informations concernant le niveau de base.

En fait, une telle réduction, si elle ne présuppose pas d'informations empiriques sur le niveau à réduire, devrait néanmoins présupposer les principes de la logique et des mathématiques. Pour cette raison, je propose d'appeler « réduction logique » le modèle de réduction attribué par Kim aux émergentistes. Une propriété résultante est réductible en ce sens logique si sa prédiction est possible sur le modèle $D-N$, mais où les seules lois invoquées sont des principes logiques et mathématiques. La prédiction de la réduction logique est une sorte bien particulière de prédiction $D-N$, à savoir une pré diction « $D-L$ » (pour « déductive-logique»), où les lois de correspondance à contenu empirique qui sont les prémisses nomologiques dans une prédiction $\mathrm{D}-\mathrm{N}$ sont absentes, pour être remplacées par des principes purement logiques et mathématiques. En ce sens, une réduction logique justifierait l'affirmation que la propriété réduite «n'est rien d'autre que » sa base de réduction, où l'on sous-entendrait par « rien » : « rien d'empirique », mais seulement la logique et les mathématiques. Une réduction logique serait en même temps une réduction ontologique dans la mesure où une théorie ne contracterait aucun engagement ontologique en faveur de propriétés ainsi réduites, pour la raison qu'elles peuvent être logiquement déduites des seules propriétés du niveau réducteur. Ainsi, le modèle fait correctement apparaître le caractère non réductionniste des doctrines dualistes mentionnées plus 
haut : ces conceptions sont incompatibles avec la réduction logique. Elles impliquent l'existence de liens nomologiques entre le mental et le physique, qui ne sont pas seulement logiques ou mathématiques.

A fin de tester la plausibilité de ce modèle, considérons la masse d'un objet complexe que Kim cite comme un cas paradigmatique de propriété « résultante » : l'information sur la masse d'un objet composé des parties $0_{1}$ et $\mathrm{O}_{2}$ semble résulter de la seule information sur les masses $m_{1}$ et $m_{2}$ des composantes $0_{1}$ et $o_{2}$. En ce sens, le calcul de la masse de l'objet complexe serait une prédiction $D-L$ : rien en dehors des règles de l'addition ne semble requis pour déduire, à partir des masses $m_{1}$ et $m_{2}$ des composantes, que la masse du composé est $m_{1}+m_{2}$. Comme $K$ im le fait remarquer dans une note, on ne considère plus aujourd'hui l'additivité de la masse comme un principe a priori, mais plutôt comme « empirique et contingent ${ }^{5}$ ». Le principe est en effet contingent car il est faux. La masse n'est additive qu'approximativement et non strictement, car dès que deux objets forment un tout par l'établissement d'un lien physico-chimique, la masse de l'ensemble est plus petite que la somme des masses, par la quantité correspondant à l'énergie de liaison.

Or si le rapport entre les masses des parties et la masse du tout est empirique (connue seulement a posteriori), Kim a tort d'affirmer que « nous pouvons expliquer pourquoi la table a cette masse à partir d'informations à propos de la masse de ses parties ${ }^{6} »$, où il est sous-entendu que cette explication ne fait appel à aucune loi ni à d'autres faits empiriques en dehors des masses des deux parties. Cela ressort del'affirmation que « la masse de cette table peut être dérivée, logiquement ou mathématiquement, de la masse de chacune de ses parties, disons le dessus de la table et sa structure ${ }^{7}{ }$. O $\mathrm{r}$ dès qu'on admet l'idée que l'additivité des masses n'est pas connue a priori, mais obéit à une loi empirique qui dépend du type de lien entre les parties lorsqu'elles sont assemblées, la dérivation de la masse du tout devient impossible sans faire appel à cette $10 i^{8}$.

5. Ibid., p. 18 note 11 .

6. Ibid., p. 18.

7. Ibid.

8. La masse $M$ d'un objet complexe est certainement une propriété basée microscopiquement (micro-based) au sens où le fait d'avoir $M$ « est la propriété d'avoir des parties propres a1, a2, ..., an, tels que P1(a1), P2 (a2),..,Pn(an), et R (a1, ..., an) » (Kim, 1997a, p. 292), où P1 est la propriété d'avoir la masse de la partie a1, etc. Rien (à l'éventuelle exception des particules élémentaires) ne peut avoir une masse $M$ sans avoir des parties qui ont une masse. $M$ ais on ne peut calculer $M$ à partir de la seule masse de ses parties. Il faut tenir compte également de l'effet nomique de la relation $R$ sur le résultat de cette « addition physique » de masses qui, en général, n'est pas simplement l'addition mathématique. Comme le dit Kim ailleurs, " la détermination n'implique, par elle-même, ni la prédictabilité ni l'explicabilité » (K im, 1997a, p. 296). O n peut interpréter cela en faisant remarquer que la détermination peut être partielle : les masses des parties déterminent partiellement, mais non totalement, la masse du tout. $M$ ais la masse du tout ne serait prédictible sur la seule base de la masse des parties que si la détermination était totale. 
Faut-il généraliser ce constat pour conclure qu'il en est ainsi de toutes les propriétés macroscopiques: qu'elles sont toutes «émergentes» au sens où elles sont " logiquement irréductibles », c'est-à-dire qu'il est impossible de prédire sur la base de la seule information sur les propriétés des parties, plus la logique et les mathématiques, la moindre propriété du tout? Pour rester avec l'exemple de la masse, il semble bien possible de prédire, sur la base de la seule logique, que le tout a une masse, même si on ne sait pas laquelle. $M$ ais en réalité, même cette prédiction bien modeste est fondée sur un principe empirique. L'interaction de certaines particules massives mène à la transformation d'une partie de leur masse en rayonnement. $N$ ous ne pouvons savoir qu'a posteriori dans quelles circonstances cela arrive et dans lesquelles cela n'arrive pas, et nous ne pouvons savoir qu'a posteriori que si une transformation de masse en énergie a lieu elle ne concerne qu'une partie de la masse, et donc qu'un tout résultant de l'interaction de deux parties d'un certain type a encore une masse quelconque, plutôt que d'avoir entièrement été transformé en rayonnement.

II n'est heureusement pas nécessaire de poursuivre plus loin la question desavoir s'il existe des macropropriétés résultantes pour montrer quela conception émergentiste de la réduction est inutilisable. Car il est difficile de trouver une propriété dont la réductibilité est moins douteuse que celle de la masse d'un objet complexe par rapport aux masses de ses parties. D onc, si la réduction logique fait de cette propriété une propriété irréductible - selon le critère énoncé plus haut de la dérivabilité sur la seule base de connaissances a priori jointes aux informations sur les parties - elle ne peut servir de modèle de la réduction telle que nous la connaissons intuitivement.

L'argument de la réalisabilité multiple montre que $\mathrm{N}$ agel avait tort de penser qu'il est nécessaire pour la réduction d'une propriété et des lois dans lesquelles elle participe qu'il existe des lois de correspondance 9 . M ais le modèle émergentiste de la réduction commet l'erreur opposée : ce n'est pas parce qu'en général il n'existe pas de loi de correspondance empirique permettant de déduire la possession d'une propriété macroscopique de la possession microscopique qu'il faut conclure que les réductions sont possibles sans aucun recours à des faits empiriques quelconques, c'est-à-dire sur la seule base de la logique et des mathématiques.

\section{La réduction fonctionnelle}

Avec la « réduction fonctionnelle $\mathrm{e}^{10} », \mathrm{Kim}$ estime avoir trouvé un modèle de réduction qui doit être préféré à celui de $\mathrm{N}$ agel et qui est une « réduction

9. Dans Kistler, 1999, le cas de la température est analysé pour montrer que la réalisabilité multiple est compatible avec la réductibilité d'une propriété.

10. Kim, 1997b, introduit l'idée de la «fonctionnalisation » d'une propriété comme étape décisive vers sa réduction. 
logique » au sens où il est conforme à l'exigence émergentiste sur la réduction : la dérivabilité logique sur la seule base des propriétés et lois du niveau de la base de réduction. $M$ ais j'essaierai de montrer que la réduction fonctionnelle n'est pas une réduction logique.

$L$ a réduction fonctionnelle comporte trois étapes : premièrement, la propriété « macroscopique » $M$ reçoit une définition fonctionnelle en termes du rôle causal qu'elle joue. Cette définition prend la forme d'une quantification existentielle sur les propriétés du niveau de base qui occupent ce rôle grâce à leurs liens causaux/nomiques à d'autres propriétés du niveau de base $^{11}$. Lors de la deuxième étape, cruciale pour notre argument, on découvre la propriété $P$ qui réalise la propriété fonctionnelle $M$ dans les individus de l'espèce $S$, où $P$ est une propriété microscopique relativement à $M$.

Comme nous le supposons dans ce cas, une réduction fonctionnelle a été accomplie, et nous supposons donc comme étant acquise la connaissance du réal isateur dela propriété $M$ dans des systèmes comme $S$, à savoir $P$ qui est une propriété ou un mécanisme au niveau de base ${ }^{12}$.

La troisième étape consiste à élaborer une théorie qui permet de comprendre comment la propriété $P$ parvient à jouer le rôle causal indiqué dans la première étape ${ }^{13}$.

II est vrai qu'il est possible, une fois les trois étapes accomplies, de prédire des occurrences de la propriété $M$, sur la base des seules informations portant sur le niveau de base. Si P est une propriété neurologique, il sera possible de prédire une occurrence de $M$ à partir de l'observation de processus neurophysiologiques qui donnent régulièrement lieu à l'occurrence de $P$. $M$ ais l'enjeu est de savoir si cette prédiction est possible sur cette seule base. $C^{\prime}$ 'est ce qu'affirme Kim lorsqu'il dit qu'après une réduction fonctionnelle « les occurrences de $M$ peuvent effectivement être prédites à partir d'informations concernant uniquement le niveau de base ${ }^{14} \gg-$ et c'est uniquement la vérité de cette affirmation qui pourrait justifier la thèse selon laquelle la réduction fonctionnelle est une réduction logique. $M$ ais cette affirmation est fausse. Examinons la deuxième étape de la réduction fonctionnelle, qui est cruciale parce que c'est là qu'est introduit le lien entre les niveaux de $M$ et de $P$. K im reconnaît que le lien entre propriété réal isatrice et propriété réalisée n'est pas d'ordre logique: « Le fait qu'une propriété neuronale donnée

11. Voir Kim, 1997b.

12. Voir Kim dans le présent numéro, p. 21

13. Cette façon de présenter les choses montre que la réduction fonctionnelle est un processus itératif : on peut concevoir la propriété $P$ à son tour comme caractérisée de manière fonctionnelle dans le réseau nomologique auquel elle prend part. Cela permet de la considérer à son tour comme une propriété macroscopique relativement à un niveau encore inférieur en taille et complexité auquel elle est susceptible d'être réduite, dès qu'elle trouve sa ou ses propriété (s) réalisatrice (s).

14.Voir Kim dans le présent numéro, p. 21. 
réalise la douleur, par exemple, est quelque chose d'empirique et de contingent ${ }^{15} »$. Faisons abstraction, dans le présent contexte, de la thèse de la contingence ${ }^{16}$. Dire que le fait que $M$ soit réalisé par $P$ dans l'espèce $S$ est empirique revient à dire que le lien de réalisation est connu a posteriori.

Pour que le lien de réalisation entre $P$ et $M$ dans $S$ puisse jouer un rôle dans l'explication et la prédiction, il faut que ce lien soit nomologique et non seulement factuel. II faut que les individus de l'espèce $S$ possèdent la propriété $P$ à chaque fois qu'ils possèdent la propriété $M$, non seulement par accident mais en vertu d'une loi, faute de quoi on ne pourrait ni expliquer ni prédire qu'un individu donné d'espèce $S$ possède $M$, à partir de la prédiction neurophysiologique qu'il possède $P$. La prédiction ou l'explication que l'individu a $M$ nécessite la prémisse nomologique selon laquelle $P$ réalise $M$ dans I'espèce $S$; autrement dit, elle nécessite une loi de correspondance locale. Ailleurs, Kim a explicitement présenté la réduction accomplie par la découverte de la propriété réalisatrice d'une propriété qui avait d'abord été caractérisée fonctionnellement, comme une « réduction locale » qui passe par « un ensemble de lois de correspondance restreintes à $S$, de la forme $S \rightarrow\left(M_{i} P_{j}\right)$, pour chaque espèce mentale $M_{i}{ }^{17}$. Lors de la réduction locale, qui est donc plus faible que l'exigence nagelienne de réduction globale, une propriété de niveau plus él evén'est pas simplement $\mathrm{N}$ agel-réduite à une propriété de niveau plus bas, mais elle est $\mathrm{N}$ agel-réduite relativement à chacun des domaines d'un nombre de domaines $\mathrm{S}$ disjoints.

Dès qu'on reconnaît l'intervention essentielle des lois de correspondance locales dans la réduction fonctionnelle, on ne peut plus la considérer comme une réduction logique ${ }^{18}$. Si les réductions fonctionnelles nécessitent des lois de correspondance locales, il n'est plus vrai que « celles-ci sont réductrices en ce sens que les ressources explicatives et prédictives qui sont mises à profit proviennent exclusivement du niveau réducteur de base ${ }^{19} »$. Kim justifie l'affirmation d'« exclusivité » en disant que :

15. Ibid., p. 24.

16. Pour montrer que la réduction fonctionnelle n'est pas une réduction logique, il suffit de reconnaître que le lien n'est pas logique et donc a priori mais empirique et donc a posteriori. En outre, il ne s'agit pas d'un simple fait contingent mais plutôt d'un lien nomologique. Je serais en désaccord avec Kim sur l'interprétation du statut modal des lois - contingentes ou nécessaires. $M$ ais il n'est pas nécessaire de trancher ce débat ici. Le point important et non controversé est que les lois ne sont connues qu'a posteriori.

17.Voir Kim, 1992, p. 21. Dans le même sens, Kim dit qu'« il suit de la définition de la réalisation que $P$ est suffisant pour $M$ - en fait, étant donné la constance nomologique [...] de la relation de réalisation, il suit que $P$ est nomologiquement suffisant pour $M$ » (K im, 1997b, p. 197).

18. Kim note explicitement que la survenance impliquée par la relation de réalisation qui est elle-même nomologique et non logique, a la force de la « nécessité nomologique (plutôt que logique/métaphysique) » (Kim, 1997b, p. 197).

19. Voir Kim dans le présent numéro, p. 21. 
C'est une définition qui nous fournit les relations réductrices entre le niveau de base et la propriété cible, en l'occurrence une caractérisation fonctionnelle de $M$ en termes de relations causales impliquant des éléments du niveau debase ${ }^{20}$.

$M$ ais la définition fonctionnelle de $M$ ne fournit qu'une quantification existentielle sur des propriétés du niveau de base qui satisfont une certaine spécification causale/nomique ${ }^{21}$. Une telle quantification existentielle ne fournit pas de lien déductif du niveau de base au niveau de $M$ dans le sens nécessaire pour la prédiction de l'occurrence de $M$, à partir des seules informations portant sur le niveau de base. L'argument suivant n'est pas déductivement valide :

(1) Fa, où a est un organisme capable d'exemplifier des propriétés mentales en exemplifiant une propriété réal isatrice du niveau de base (neurophysiologique), et $F$ est une propriété du niveau de base.

(2) $(\forall x)(M x(\exists P)(P x \& D(P))$, où $D$ est une condition causale/nomique sur les propriétés $\mathrm{P}$ du niveau de base ${ }^{22}$.

Donc, $\mathrm{Ma}$.

Il est vrai que (2), la définition fonctionnelle de $M$, fournit un lien entre les deux niveaux de propriétés, et même un «lien déductif », mais en I'absence d'information sur lefait que la propriété $F$ du niveau de base figure parmi les réalisatrices de $M$, il est impossible de déduire l'occurrence de $M$ à partir d'information sur l'occurrence de F. Il est également vrai qu'il n'est pas nécessaire, pour prédire l'exemplification de $\mathrm{M}$ par a, de connaître la propriété particulière qui réalise $M$ dans les organismes du type de $a$. II suffit de compléter les prémisses (1) et (2) ou bien par l'ensemble des propriétés réalisatrices de $M$ (parmi lesquelles figure $F$ ), ou bien par un sous-ensemble de ces réalisatrices comprenant $F$. $M$ ais en tout état de cause, la seule quantification existentielle figurant dans la définition fonctionnelle (2) n'y suffit pas.

$N$ otre thèse selon laquelle la deuxième étape d'une réduction fonctionnelle mobilise une relation de réalisation nomologique (une loi de correspondance locale ${ }^{23}$ ) est plus forte qu'il n'est nécessaire pour le présent argument qui vise à montrer que la réduction fonctionnelle n'est pas une réduction logique : pour cela, il suffit de montrer que le lien de réalisation entre $M$ et $P$ dans $\mathrm{S}$ est un fait empirique. O r, comme nous l'avons noté plus haut, cela est explicitement reconnu par $\mathrm{Kim}^{24}$.

20. Ibid., p. 21.

21. Voir Kim, 1997b, p. 195.

22. Cette équivalence correspond à la conception fonctionnaliste des propriétés mentales comme propriétés de second ordre, et plus précisément des « propriétés fonctionnelles » qui sont les « propriétés de second ordre sur B [soit une classe de propriétés non mentales du niveau de base, qui inclut des propriétés physico-chimiques, biologiques et comportementales] dont la spécification D implique la relation causale/nomique» (Kim, 1997b, p. 194-195).

23. Cette thèse peut s'appuyer sur certaines suggestions de Kim ; voir Kim, 1992 et 1997b. 24. Voir la note 15. 
Kim nie l'importance de la deuxième étape de la réduction fonctionnelle qui, selon notre analyse, est cruciale et qui consiste à trouver la propriété réalisatrice $P$ d'une propriété $M$, pour un système de type $S$. Pour Kim « la question cruciale » est de savoir si la propriété est « fonctionnalisable », ce qui exige qu'elle puisse recevoir une définition implicite fonctionnelle et que son rôle fonctionnel soit rempli par une propriété réalisatrice quelconque. Et, comme il le note, « si une propriété mentale a été fonctionnalisée, le reste de la tâche revient à la science et, d'un point de vue métaphysique, c'est comme si elle avait été réduite ${ }^{25} »$. O $r$, si notre analyse est correcte, la fonctionnalisation seule ne suffit pas pour l'explication ou la prédiction d'une occurrence de $M$ : il faut en outre connaître la loi de correspondance locale

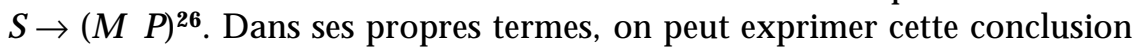
ainsi : «La possibilité de la fonctionnalisation [...] est une condition nécessaire de la microréduction », mais elle n'est pas suffisante, car « la question de savoir si la réduction sera actuellement accomplie est une question empirique [...] de la science ${ }^{27} »$. La réduction fonctionnelle, c'est-à-dire l'indication d'une définition fonctionnelle, ou fonctionnalisation, ne mène qu'à la réductibilitée ${ }^{28}$, mais non à la réduction, et en particulier pas à la réduction logique.

Par conséquent, I'argument par lequel Kim montre que la réduction nagelienne n'est pas une réduction logique s'applique mutatis mutandis à la réduction fonctionnelle qui est elle aussi nagelienne, quoique seulement de façon locale. À la place de la prédiction nagelienne suivante :

« Le système $\mathrm{S}$ exemplifiera $\mathrm{T}$ à $\mathrm{t}$

M T

Donc, S exemplifiera M à t29 »,

où « $M T$ » est uneloi de correspondance globale, la réduction fonctionnelle fournit la prédiction suivante:

Le système $\mathrm{S}$ exemplifiera $\mathrm{T}$ à $\mathrm{t}$

$\mathrm{S} \rightarrow(\mathrm{M} \mathrm{T})$

Donc, S exemplifiera M à t »,

25. Voir Kim le présent numéro, p. 24.

26. Ce que la fonctionnalisation établit à elle seule, c'est que la propriété fonctionnalisée est causalement efficace, en héritant des pouvoirs causaux de ses propriétés réalisatrices ; voir Kim, 1997a, p. 295. Cela s'explique du fait que fonctionnaliser $M$ signifie définir $M$ en termes des relations causales dans lesquelles entrent les objets qui possèdent $M$, en vertu du pouvoir causal de l'une de leurs propriétés réalisatrices.

27. Voir Kim, 1997b, p. 202-203.

28. «La conception fonctionnaliste des propriétés mentales est [...] nécessaire et suffisante pour la réductibilité » (Kim, 1997b, p. 203).

29. Voir Kim dans le présent numéro, p. 22. 
où « $S \rightarrow(M T)$ » est une loi de correspondance locale qui dit que dans les systèmes $S, M$ est réalisée par $T$.

O n peut donc retourner la critique kimienne de la réduction nagelienne contre sa propre réduction fonctionnelle. La citation suivante de K im s'applique aussi bien aux prédictions du second type qu'à celles du premier type : «De telles prédictions d'occurrences de $M$ ne sont donc pas fondées uniquement sur la base d'informations concernant le phénomène et les lois au niveau de base et, par conséquent, elles ne satisfont pas le desideratum sur la réduction ${ }^{30} »$, c'est-à-dire la réduction logique.

On peut également retourner contre Kim la critique qu'il adresse à la théorie selon laquelle les propriétés mentales sont identiques à des propriétés cérébrales à la manière des identités nécessaires mais a posteriori de K ripke, sur le modèle de l'identité eau $=\mathrm{H}_{2} \mathrm{O}$, à savoir :

Comme ces identités [...] ne peuvent être connues qu'empiriquement, et comme elles sont requises pour toute prédiction d'occurrences mentales reposant sur de l'information relative aux neurones, par exemple pour la prédiction d'occurrences de la douleur, il ne sera pas possible de prédire de telles occurrences uniquement sur la base d'information neurobiologique ou behaviorale à propos des organismes ${ }^{31}$.

Étant donné que le lien factuel (et nomologique) entre la propriété fonctionnelle $M$ et sa réalisatrice dans un individu de l'espèce $S$ ne peut lui aussi être connu qu'empiriquement, et que la connaissance de ce lien est indispensable à la prédiction d'une occurrence de $M$ à partir d'informations portant sur le niveau de base, cette prédiction n'est pas non plus conforme aux exigences de la réduction logique.

\section{Conclusion}

La doctrine émergentiste cherche à rendre justice à l'intuition sel on laquelle la satisfaction des contraintes nageliennes sur la réduction, loin de suffire à une réduction achevée, ne permet au mieux que de poser clairement la question suivante, à laquelle la réduction répondrait : pourquoi la propriété $M$ est-elle nomologiquement liée, dans les individus del'espèce $S$, à la propriété réalisatrice $P$ ? Considérer $M$ comme une propriété émergente, aussi longtemps qu'aucune réponse n'est trouvée et que ce lien reste un fait brut inexpliqué, c'est donner expression à l'intuition quel'existence de $M$ est, dans ces conditions, inintelligible du point de vue physicaliste. Kim propose la réduction fonctionnelle comme un modèle de réduction qui ne permet plus seulement de poser la question mais d'y répondre. Donner une définition fonctionnelle de la propriété $M$, c'est la caractériser en termes de relations

30. Ibid.

31. I bid., p. 24. 
causales/nomiques que les objets qui possèdent $M$ entretiennent grâce à leur possession de l'une ou l'autre des propriétés réalisatrices de $M$. Comme la propriété réalisatrice est mentionnée dans la définition même de $M$, le fossé entre le niveau réalisateur et le niveau réalisé semble être comblé par un lien conceptuel et donc a priori intelligible. Par conséquent, Kim affirme avoir trouvé, avec la réduction fonctionnelle, une forme de réduction qui répond à l'exigence émergentiste de rendre lelien entrela propriété $M$ et sa propriété réalisatrice intelligible, plutôt que de le laisser à l'état de fait brut mystérieux comme le fait la réduction nagelienne.

$M$ ais, j'ai cherché à montrer que la réduction fonctionnelle permet d'expliquer ou de prédire une occurrence de $M$ uniquement grâce à l'introduction d'un nouveau fait brut qui, du point de vue émergentiste, est tout aussi mystérieux qu'une loi de correspondance nagelienne qui, tout comme cette dernière, est un fait nomologique et qui dépasse la seule définition fonctionnelle ; il s'agit du fait empirique que $M$ possède les propriétés réalisatrices $P_{1}, P_{2}, \ldots P_{n}$. La question $n$ 'est plus : pourquoi $M$ est-elle liée à $P$ par une loi de correspondance? mais plutôt : pourquoi $M$ est-elle réalisée par $P_{1}$ ou $P_{2}$ ou ... $P_{n}$ ? M ais il n'y a de réponse purement logique ou mathématique ni à l'une ni à l'autre de ces questions, et en ce sens, selon le critère émergentiste, $M$ resterait toujours une propriété émergente même après une réduction fonctionnelle.

II n'y a pas lieu de contester l'intuition qui supporte le critère émergentiste de la réduction logique. II semble parfaitement légitime de trouver qu'il existe un sens dans lequel même la découverte du réalisateur $P$ de la douleur dans les humains ne nous permet toujours pas de comprendre d'où vient la douleur, sur la base de la connaissance de $P$ et des processus qui mènent aux occurrences de P. A près tout, comme j'ai essayé de le montrer, la seule connaissance du niveau de base ne suffit pas pour déduire logiquement une occurrence de douleur. $M$ ais la considération du cas de la masse d'objets complexes plaide contre l'interprétation émergentiste de la réduction adoptée par K im, c'est-à-dire contre l'idée d'identifier les propriétés émergentes et irréductibles et les propriétés résultantes et réductibles. La masse d'un objet complexe ne se laisse pas déduire logiquement ou mathématiquement à partir de la seule information sur ses parties et leurs masses, sans information sur la loi de correspondance qui spécifie la masse du tout étant donné les masses des parties et la nature de leur liaison. Le concept émergentiste de « réduction logique » fait de la masse du tout une propriété irréductible (car émergente) à la masse des parties. $M$ ais un concept de réduction qui mène à considérer comme irréductible une propriété aussi clairement réductible que la masse d'un objet complexe est trop étroit. 


\section{Bibliographie}

Kim, J., 1992. «M ultiple Realization and the M etaphysics of Reduction », Philosophy and Phenomenological Research, 52, p. 1-26; réédité dans Kim, J., Supervenience and $M$ ind, Cambridge ( $M$ ass.), Cambridge University Press, 1993, chap. 16.

Kim, J., 1997a. "Does the Problem of M ental Causation Generalize? », Proceedings of the Aristotelian Society, 97, p. 281-297.

Kim, J., 1997b. "The M ind-Body Problem: Taking Stock after Forty Years », dans Tomberlin, J, dir., Philosophical Perspectives 11, M ind, Causation, and World, Boston, Basil Blackwell, p. 185-207.

Kim, J., 2000. "Émergence, modèles de réduction et le mental », Philosophiques, 27, $n^{\circ} 1$, p. 9-24.

Kistler, M., 1999. "Multiple Realization, Reduction, and M ental Properties », International Studies in the Philosophy of Science, 13, $n^{\circ} 2$, p. 135-149.

N agel, E., 1961. The Structure of Science, London, Routledge and Kegan Paul. 